A Practical Data Collection Tool for Mass-Casualty Incidents and Disasters: The Medicina de Emergencia Basada en la Evidencia (MEBE) Datasheet

Alfredo Serrano-Moraza, ${ }^{1}$ M.J. Brinas-Freire, ${ }^{1}$ A. PachecoRodgriguez, ${ }^{2}$ A. Pérez-Belleboni ${ }^{3}$

1. SUMMA-112, Madrid, Spain

2. Emergencia Ciudad Real ECR-SESCAM Helicsa 112, CastillaLa Mancha, Spain

3. SAMUR, Madrid, Spain

Introduction: Randomized, prospective clinical trials are neither ethical nor possible in mass-casualty incidents (MCI) and disasters, and exercises and computer simulations have limited validity. Therefore, much of the evidence used in emergency planning and preparedness necessarily arises from retrospectively collected data from events that have actually occurred.

Objective: This presentation describes the development of the Medicina de Emergencia Basada en la Evidencia (MEBE) or Evidence-Based Emergency Medicine datasheet, a practical data collection tool for MCIs and disasters.

Methods: Descriptive information was obtained from: (1) observations; (2) an Internet search using an advanced Google interface and selected key words, such as "mass-casualty incident", "disaster medicine", "evaluation", "analysis", "research"; (3) an online search using a MEDLINE interface and selected medical subject headings; (4) manual searches of identified references; and (5) expert consultation via mail, phone, and personal interviews.

Results: Development of the MEBE datasheet began after the 2003 Madrid bombings. Online and manual searches identified no useful data collection tools for $\mathrm{MCIs}$ or disasters. A working group of expert participants in MEBE suggested that many disasters may be divided into component MCIs, facilitating their analysis and evaluation. The working group also suggested relevant data elements for inclusion in the MEBE datasheet, emphasizing those elements that impacted mortality. These data elements were organized into modules on a single-page electronic datasheet, using Microsoft Access software. Elements of the MEBE datasheet include: (1) basic event information (type, date); (2) on-scene triage (occurrence, method, results); (3) number of injured patients and injury severity (dead, critically injured, severely injured, lightly injured); (4) prehospital resources; (5) special rescue or chemical, biological, radiological, and nuclear needs; (6) ambulation evacuation index; (7) number of general hospitals and distance from scene; and (8) time.

Potential advantages of using the MEBE datasheet include: (1) it facilitates the systematic collection of information about MCIs and disasters; (2) it aids the evaluation of emergency response during MCIs and disasters; (3) it may be used as the basis for organizing all available evidence about $\mathrm{MCI}$ s and disasters for future emergency planning; and (4) it may be posted online in order to share useful results (http://www.mebe.org).
Conclusion: The MEBE datasheet is a systematic, modular data collection tool, which may facilitate data sharing and communication about MCIs and disasters.

Keywords: data collection; mass-casualty incident; preparedness Prehosp Disast Med 2005;20(5):s166.

\section{The Regional Medical Operations Center: Lessons Learned from South Central Texas Rasa Silenas}

Office of Homeland Security, Texas A\&M University Health Science Center, Brooks City Base, Texas USA

Introduction: Large-scale, health emergencies, including those caused by natural disasters, emerging infectious diseases, transportation and industrial accidents, and terrorism easily can transcend the health resources of a community, and have repercussions throughout surrounding areas. A major challenge for medical disaster response is that healthcare resources, unlike fire services and law enforcement agencies, are largely non-governmental and report to a diverse set of employers.

Objective: This presentation describes the organization of a regional emergency medical operations center (RMOC) in south central Texas.

Results: The region targeted for the RMOC was Trauma Service Area P, a 22-county area that encompasses urban, rural, border, and coastal communities. Although this region is aligned with patient flows, it does not correlate with State Disaster Districts. The RMOC successfully links public health agencies, general and specialty hospitals, and professional associations for medical disaster response in this region. Success factors included: (1) aggressive recruitment of representatives from throughout the region; (2) the generosity of key stakeholders in providing funding, space, and personnel support; (3) meticulous transparency in planning and funding; (4) a well-conceived supporting software infrastructure; and (5) the availability of administrative staff. Pitfalls include: (1) the difficulty of documenting rapidly evolving plans and procedures; (2) a dependency on a small number of key individuals; and (3) jurisdictional barriers.

Conclusion: While far from mature, the RMOC has won the ongoing support of key stakeholders, and promises to be a valuable addition to regional medical disaster response capabilities.

Keywords: barriers; emergencies; funding; hospitals; planning; public health agencies; Regional Medical Operations Center (RMOC); stakeholders

Prebosp Disast Med 2005;20(5):s166 